


\title{
Der Weg des Virus
}

\author{
Medizinisch gesicherte Erkenntnisse "Corona" ist derzeit in aller Munde und \\ beherrschendes Thema in den Medien. Doch trotz oder gerade wegen der Viel- \\ zahl der sich teils widersprechenden Nachrichten sind viele Zusammenhänge \\ unklar. Daher versuchen wir, die nach aktuellem Stand medizinisch gesicherten \\ Erkenntnisse zusammenzufassen.
}

$\mathbf{U}$ $m$ die Gefahr von Infektionskrankheiten besser einschätzen zu können, muss man verstehen, wie der menschliche Körper mit Erregern umgeht. Darüber hinaus ist von Bedeutung, die Ausbreitung innerhalb der Bevölkerung zu verfolgen.

\begin{abstract}
Abwehr von Erregern
Neben Bakterien gehören Viren zu den klassischen Erregern für Infektionskrankheiten, insbesondere auch für Erkältungs- und Atemwegserkrankungen. In ihren biologischen Eigenschaften unterscheiden sie sich jedoch deutlich von Bakterien (Tab. 1). Im menschlichen Körper gibt es mehrere Abwehr-Mechanismen. Die unspezifische Abwehr ist immer präsent und bildet eine erste Barriere gegen eindringende Keime und Partikel. Sie finden sich an allen Eingangsstellen des Körpers. Dazu gehören die Zilien des Atemtraktes oder Sekrete wie Speichel und Tränenflüssigkeit. Doch dies genügt bei einem Erstkontakt mit den neuen Erregern nicht, um den Körper gänzlich vor einer Infektion schützen. Daher können alle Schutzmaßnahmen helfen, die das Eindringen der Erreger über die Schleimhäute verhindern.
\end{abstract}

\section{- Je mehr neue Viren entstehen und Zellen zerstört werden, desto deutlicher merkt der Mensch die Symptome.}

Wissenschaftler am Massachusetts Institute of Technology (USA) haben herausgefunden, dass beim Niesen virenhaltige Tröpfchenwolken mit einer Geschwindigkeit von bis zu $150 \mathrm{~km} / \mathrm{h}$ explosionsartig in die Luft geschleudert werden. Die Tröpfchen sind unterschiedlich groß und können mehrere Meter weit fliegen. Sie können direkt auf Menschen treffen oder sich auf Oberflächen ablagern. Erste Studien haben gezeigt, dass das neue Coronavirus dort bis zu mehreren Tagen überlebensfähig ist. Hier wird die Bedeutung der zwei Hauptmaßnahmen deutlich: Zum einen sollte durch gute Niesetikette (Taschentuch oder Ellenbeuge) eine Flugwolke vermieden werden, zum anderen kann Abstand helfen, nicht von den Tröpfchen direkt getroffen zu werden und diese einzuatmen. Andererseits sind die Hände die Hauptvehikel, die Erreger auf
Oberflächen und Gegenständen aufnehmen und durch unbedachtes Berühren auf die Schleimhäute tragen, von wo die Erreger in den Körper eindringen können. SARS-CoV-2 zählen zu den behüllten Viren, d.h. sie sind von einem Lipidmantel umgeben. Wenn diese Lipidhülle z.B. durch gründliches Waschen mit Seife oder Desinfektionsmittel zerstört wird, sind die Viren nicht mehr lebensfähig. Daher ist das strikte Einhalten der Händehygiene-Regeln äußerst wichtig. Da noch keine genauen Zahlen vorliegen, wird nicht ausgeschlossen, dass je nach Oberfläche das SARS-CoV-2-Virus mehrere Stunden bis Tage außerhalb des Körpers überlebt. Im Vergleich dazu kann das HI-Virus nur wenige Minuten auf Oberflächen überstehen.

\section{Das Schlüssel-Schloss-Prinzip}

Wenn sie erst einmal im Körper sind, dringen die Viruspartikel mit Hilfe bestimmter Oberflächenmoleküle nach einem SchlüsselSchloss-Prinzip in die Zielzellen ein. So tragen Hepatitis-Viren bestimmte Strukturen, die genau auf menschliche Leberzellen passen und so in sie eindringen können. Erkältungs-, Grippe- und Corona-Viren finden in der Regel über Zellen des Atemtrakts ihren Weg in den Körper. Im Fall der Coronaviren sind es die namensgebenden „Krönchen“, die an die Zielzellen andocken.. Hier erklärt sich auch, warum Raucher, Asthma- oder COPD-Patienten besonders gefährdet sind: Ihr Atemtrakt ist bereits vorgeschädigt und anfälliger für die Erreger.

Die Viren vermehren sich in den befallenen Zellen und zerstören diese dabei. Je mehr neue Viren entstehen und Zellen zerstört werden, desto deutlicher merkt der Mensch die Symptome. Diese entstehen zum Teil durch die zerstörten Zellen, zum anderen aber auch durch weitere unspezifische Abwehrmaßnahmen des Körpers (Entzündungsreaktion), wie Fieber. Manche Medikamente unterdrücken diese Entzündungsreaktion und schwächen dadurch die Infektabwehr. Zu den bekanntesten dieser Medikamente gehören die Glukokortikoide (Kortison), das bei ungewollten Immunreaktionen wie Asthma, Lupus erythematodes oder rheumatischen Erkrankungen eingesetzt wird.

Die Zeit vom Eindringen der Erreger bis zum Ausbruch von Krankheitszeichen nennt man Inkubationszeit. Dabei kann der Betroffene schon Erreger weitergeben, bevor er merkt bzw. vermutet, 


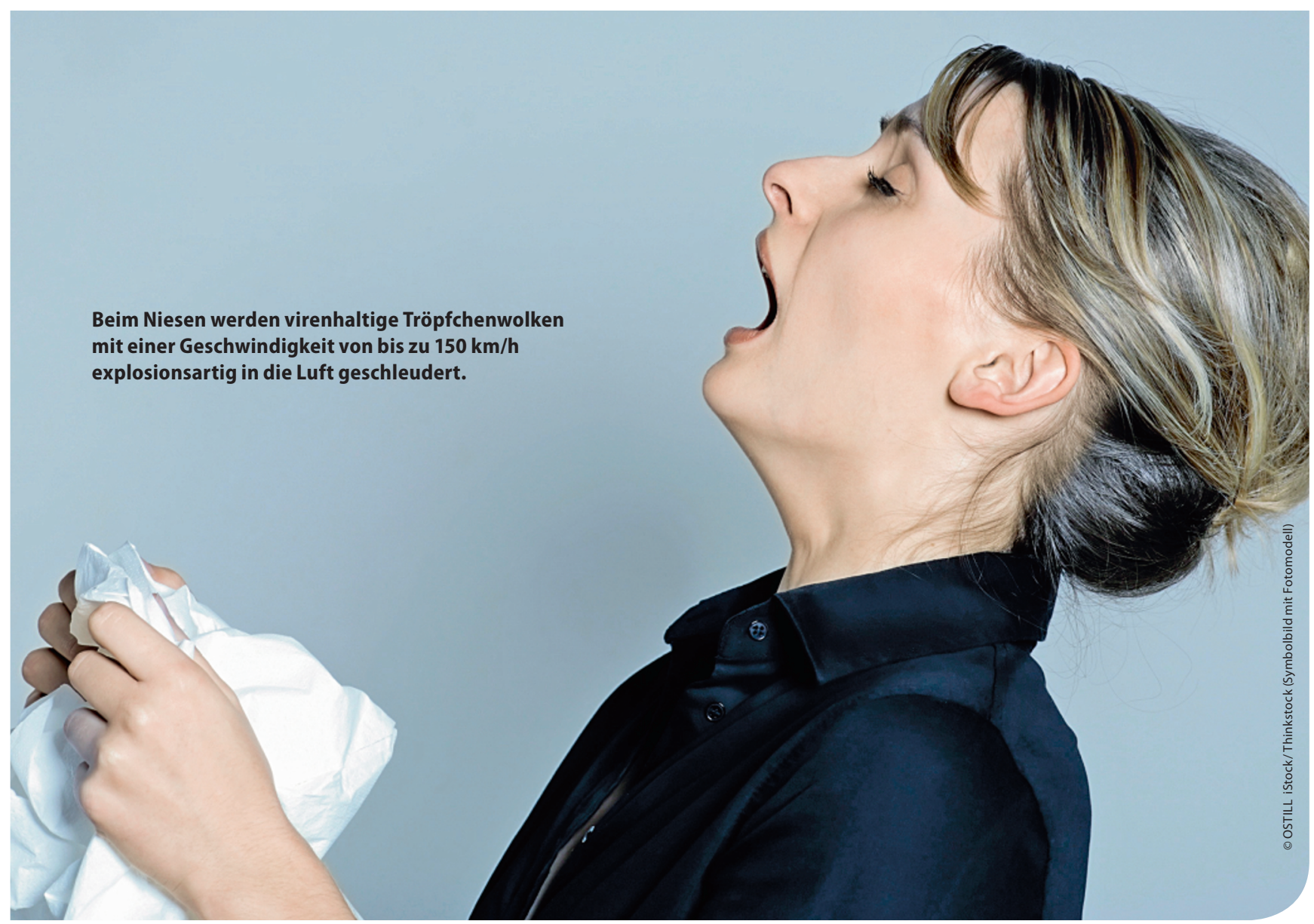

dass er infiziert ist. Die Inkubationszeit ist abhängig vom Erreger, kann aber auch individuell unterschiedlich sein. So werden bei der aktuellen COVID-19-Erkrankung Inkubationszeiten von ein bis 14 Tagen berichtet, wobei die meisten Patienten nach etwa fünf Tagen die ersten Symptome zeigen. Der „Kampf“ des Körpers wird durch die Symptome deutlich. Parallel zur unspezifischen Abwehr beginnt der Körper bereits damit, die spezifische Abwehr aufzubauen. Sie ist hocheffizient, benötigt aber Zeit, bis sie bereit ist. Je stärker das körpereigene Immunsystem ist, desto schneller ist die spezifische Abwehr bereit und desto milder ist der Krankheitsverlauf und kürzer die Dauer der Erkrankung. Bei Patienten nach Transplantation wird das Immunsystem absichtlich geschwächt, damit es nicht zur Abstoßung des neuen Organs kommt.

\section{Hilfe von außen}

Die spezifische Abwehr besteht aus T-Lymphozyten und im Blut zirkulierenden Antikörpern, die die Erreger erkennen und eliminieren. Sie passen wiederum wie Schlüssel und Schloss auf die „Krönchen“ der SARS-CoV-2 Viren. Die Symptome verschwinden, der Patient genest, was bei den meisten COVID-19 Patienten nach etwa zwölf Tagen der Fall ist. Bei Menschen, deren Immunabwehr jedoch geschwächt ist, dauert es länger, bis die spezifische Immunabwehr anläuft und es werden immer mehr Wirtszellen zerstört. Im Falle der aktuellen Coronaviren bedeutet dies, dass immer mehr Lungengewebe geschädigt ist, was sich in Form einer schweren, atypischen Pneumonie äußert, die im CT ein typisches Bild zeigt. Daher kann ein längerer Krankheitsverlauf auf Komplikationen und die Gefahr von Lungenversagen hindeuten.

Für bestimmte Krankheiten (z.B. Tetanus), können Antikörper direkt verabreicht werden, eine sogenannte passive Immunisierung. Damit hat der Patient einen Sofortschutz, ohne dass er eine Infektion durchmachen musste. Allerdings besteht kein dauerhafter Schutz (nur etwa drei Monate), da die körpereigene Abwehr nicht stimuliert wurde. Aktuell arbeitet die Medizinische Hochschule Hannover daran, aus Plasmaspenden von genesenen Patienten Antikörper zu filtrieren, um sie schwerkranken COVID-19 Patienten zu transfundieren. Um sicher zu gehen, dass dabei aber nicht auch das Virus übertragen wird, müssen die Patienten bereits seit mindestens vier Wochen wieder gesund sein. Ein effektiver Langzeitschutz kann nur durch eine aktive Impfung (Immunisierung) erreicht werden. Dabei werden abgetötete oder abgeschwächte Erreger geimpft, die zur Stimulierung der körpereignen Abwehr führen, ohne dass man daran erkrankt. Derzeit werden bereits mehrere Impfstoffe im Labor getestet, seit Anfang April laufen erste klinische Studien.

\section{Der Faktor Zeit}

Je langsamer sich also die Krankheit ausbreitet, desto mehr Zeit haben Ärzte und Forscher für die Entwicklung von Therapien und Impfstoffen. In diesem Zusammenhang wird immer wieder die Reproduktionszahl erwähnt. Dieser Begriff der Epidemiologie gibt die Zahl der Zweitinfektionen an, die von einem Fall ausgehen. Zu Beginn des Ausbruchs in Deutschland lag diese Zahl bei etwa drei. Das bedeutet, dass von einem Infizierten drei weitere Menschen

\section{- Die Reproduktionszahl gibt die Zahl der Zweitinfektionen an, die von einem Fall ausgehen.}

angesteckt werden, was zu einem exponentiellen Anstieg von Krankheitsfällen in kürzester Zeit führt. Das heißt, dass zwei Drittel der Fälle vermieden werden müssten, um den Ausbruch unter Kontrolle zu bekommen. Die Reproduktionszahl stellt jedoch einen Mittelwert aus allen Bevölkerungsteilen dar (Stand 08.04.2020). 


\section{PFLEGE KOLLEG}

\section{Tab. 1: Unterschiede von Viren und Bakterien}

\begin{tabular}{|l|l|l}
\hline & Virus & Bakterien \\
\hline & "Dem Leben nahe stehend" & Lebewesen \\
\hline Aufbau & Partikel (keine Zellen) aus Erbmaterial und Kapsid & Zellen mit Zellmembran, aber ohne Zellkern oder Organellen \\
\hline Erbmaterial & DNA oder RNA & DNA und RNA \\
\hline Umhüllung & Mit oder ohne Hülle & Membran und (meist) Zellwand \\
\hline Stoffwechsel & Kein eigener Stoffwechsel & Stoffwechsel \\
\hline Vermehrung durch .... & $\begin{array}{l}\text { Eindringen über Bindestellen, Nutzung und Zerstörung der } \\
\text { Wirtszellen }\end{array}$ & Eigenständige Teilung \\
\hline Größe & $\begin{array}{l}\text { variabel, } \\
\text { z.B. SARS-CoV-2 ca. 125 nm (etwa mehr als 1/100 mm) }\end{array}$ & $\begin{array}{l}\text { Sehr variabel, } \\
\text { z.B. S. aureus ca. 1 } 1 \mu m \text { (ca. 1/10 mm) }\end{array}$ \\
\hline Typische Krankheiten & $\begin{array}{l}\text { Influenza, Norovirengastroenteritis, AIDS, Masern, FSME, SARS, } \\
\text { MERS }\end{array}$ & Meningokokken-Meningitis, Salmonellen, Scharlach \\
\hline Medikamente & (teilweise) Virostatika & Antibiotika
\end{tabular}

\section{Buchempfehlung}

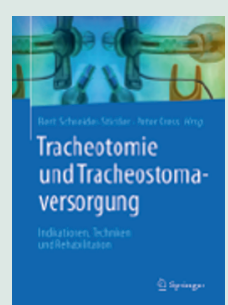

Tracheotomie und Tracheostomaversorgung Indikationen, Techniken \& Rehabilitation

Berit Schneider-Stickler, Peter Kress (Hrsg.)

1. Aufl. 2018, XX, $378 \mathrm{~S}$

$273 \mathrm{Abb}$. in Farbe.

Softcover: ISBN 978-3-7091-4867-9

$56,53 €(A)$

eBook: ISBN 978-3-7091-4868-6 $42,99 €$

Die Tracheotomie ist auf Intensivstationen eine der häufigsten Interventionen. In der Kopf-HalsChirurgie sind anspruchsvolle Eingriffe oft nicht ohne Tracheotomie möglich. „Can't intubate can't ventilate"-Situationen erfordern rasches ärztliches Handeln zur Sicherung der Atemwege: entweder die notfallmäßige Koniotomie oder chirurgische bzw. perkutan-dilatative Tracheotomie-Techniken.

Das postoperative Tracheostoma-Management erfordert eine hochspezialisierte Wundversorgung und fachgerechte Nachsorge. Es war ein besonderes Anliegen, Standards zur Tracheostomaversorgung vorzustellen und auf Kanülensysteme und Tracheostomazubehör einzugehen. Ein besonderer Stellenwert wird der Stimm-, Sprach- und Schluckrehabilitation nach Tracheotomie beigemessen.
Dabei sind jedoch nicht alle Menschen gleich beteiligt. Je mehr Kontakte ein Mensch hat, desto mehr Übertragungsmöglichkeiten gibt es. So sind zum Beispiel in einer Klasse mit 25 Schülern theoretisch von einem Fall ausgehend 24 Neuinfektionen möglich, die wiederum in die Familien oder in Freizeit und Sportgruppen getragen werden können. Von dort sind wiederum viele weitere Übertragungen an Freunde oder Arbeitskollegen möglich. Insbesondere bei jüngeren Kindern ist die Einhaltung der Händehygiene und Atemetikette nicht immer gewährleistet. Hier setzen die Schutzmaßnahmen der Schulschließungen und des Social Distancing an, um die Ausbreitung zu verlangsamen.

Dr. Andrea Jessen

\section{: PFLEGE EINFACH MACHEN}

Menschen können Krankheiten übertragen, bevor sie wissen, dass sie infiziert sind. Deshalb: Schutzmaßnahmen wie Händehygiene, Atemetikette und Abstand immer einhalten.

Symptome treten erst durch die Abwehrreaktion des Körpers auf, wenn Zellen durch die Viren zerstört werden und die Infektion schon fortgeschritten ist. Die Dauer und Ausprägung der Symptome hängt vom Immunsystem des Erkrankten ab. Die Gabe von Antikörpern bietet einen Sofortschutz, während die aktive Immunisierung einen Langzeitschutz bietet.

Solange kein effektiver Schutz oder Therapie vor einer SARSCoV2-Infektion besteht, sind Schutzmaßnahmen, die die Ausbreitung reduzieren und verlangsamen, der wichtigste Schutz vor der Krankheit. Sie schützen auch vor anderen Krankheiten mit gleichem Übertragungsweg, z.B. Influenza. 


\title{
Corona, COVID-19, SARS-CoV-2
}

\author{
Diese Regeln gelten jetzt In den letzten 20 Jahren waren Coronoaviren ursäch- \\ lich für drei große Ausbrüche mit schweren Lungenerkrankungen: SARS, MERS \\ und jetzt aktuell COVID-19. COVID-19 verbreitet sich aber deutlich rasanter als die \\ Vorgänger. Welche Eigenschaften besitzen Corona-Viren? Wie können sich Pfle- \\ gende effektiv vor SARS-CoV-2 schützen? Und welche Regeln gelten in Kliniken, \\ Pflegeheimen und für ambulante Dienste?
}

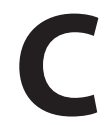
oronaviren bilden eine Gruppe mit mehreren Hundert verschiedenen Viren, deren natürliche Wirte Tiere sind (z.B. Schweine, Kamele, Katzen, Fledermäuse), die jedoch in seltenen Fällen auf den Menschen überspringen können. Dort lösen sie meist milde, erkältungsähnliche Erkrankungen oder Bronchitis aus (Tab. 1). Der Vergleich zeigt, dass zwar die Sterblichkeit bei SARS und MERS deutlich höher lag, jedoch ist die Verbreitung von COVID-19 deutlich rasanter mit vielfach höheren Erkrankungszahlen. Hier wird auch die Gefahr durch Überlastung für das Gesundheitswesen deutlich: Bei einem explosiven Anstieg ohne Schutzmaßnahmen würde es zum Zusammenbruch der Gesundheitsversorgung und der Beatmungsplätze kommen, was wiederum zu einer erhöhten Sterblichkeit führen würde. Daher sollen die aktuellen Maßnahmen einen „Flächenbrand“ verhindern und die Erkrankungszahlen zeitlich entzerren.

\section{Bei schwerem Verlauf kann sich eine atypische Lungenentzündung bis hin zur Sepsis entwickeln, sodass der Patient intensiv- und beatmungspflichtig wird.}

\section{Symptome bei COVID-19}

$\mathrm{Zu}$ den typischen Symptomen einer Erkrankung zählen Fieber, Husten, Erschöpfung und Erkältungszeichen. Bei etwa einem Drittel der Erkrankten kommt es zu Diarrhoen, ebenso wird häufig über Geruchs- und Geschmacksverlust berichtet. Bei schwerem Verlauf kann sich eine atypische Lungenentzündung bis hin zur Sepsis entwickeln, sodass der Patient intensiv- und beatmungspflichtig wird. Dies entwickelt sich schleichend, sodass ein längerer Krankheitsverlauf erhöhte Wachsamkeit erfordert. Zu den Risikopatienten zählen Lebensalter >60 Jahre, Menschen mit Herz-Kreislauf-, Leber- und Nierenerkrankungen, Immunschwäche (Transplantationspatienten, Krebserkrankungen, AIDS) und Multimorbidität. Schwangere gelten derzeit nicht als Risikogruppe. Kinder und Jugendliche erkranken fast nicht, können jedoch Überträger sein.

\section{Hygienemaßnahmen und Konsequenzen für die Pflege}

Nach aktuellen Informationen des Robert Koch-Instituts (RKI) spielt bei der Übertragung des SARS-CoV-2 die Beteiligung der oberen Atemwege eine eminente Rolle. Meist erfolgt die Übertragung direkt über Atemwegsekrete, die bei Husten oder Niesen in Form von Tröpfchen freigesetzt werden, aber auch bei bestimmten medizinischen Eingriffen, bei denen es zur Aerosolbildung kommen kann (z.B. Bronchoskopie) oder indirekt über Hände und/oder kontaminierte Flächen. Eine Übertragung durch Kontakt mit erregerhaltigen Ausscheidungen kann derzeit nicht ausgeschlossen werden.

Da es bisher keine wirksame, erregerspezifische Therapie oder Impfung gibt und die Behandlung symptomatisch erfolgt, ist Übertragungsvermeidung besonders wichtig. Auf der Basis von SARS und MERS sowie den aktuellen Erkenntnissen hat das RKI folgende Empfehlungen erarbeitet:

- Konsequente Umsetzung der bestehenden Basishygiene

- Striktes Einhalten der Händehygiene. Machen Sie sich die fünf Momente der Händehygiene (für Desinfektion bzw. Handschuhwechsel) bewusst: Vor und nach jedem Patientenkontakt oder nach Kontakt mit der Patientenumgebung. Vor aseptischen Tätigkeiten und nach Kontakt mit potenziell kontaminiertem Material (z.B. Taschentücher)

- Vermeidung von Verbreitung durch Bedeckung von Mund und Nase bei Niesen, Schnäuzen, Husten mit einem Taschentuch oder notfalls der Ellenbeuge (gute Atemetikette)

- Benutzte Taschentücher sofort entsorgen

- Abstand halten von Personen, die niesen, husten oder schnäuzen

- Vermeiden von Berührung der Schleimhäute an Augen, Nase und Mund mit den Händen

\section{Maßnahmen im Umgang mit COVID-19 Patienten}

\section{Räumlichkeiten:}

_ Einzelzimmer mit Nasszelle, vorzugsweise Isolierzimmer mit Schleuse

- Kohortenisolierung (Gruppenisolierung) mehrerer COVID-19 Patienten nur unter ganz bestimmten Voraussetzungen (z.B. 


\section{PFLEGE KOLLEG}

Tab. 1: Vergleich von bekannten Ausbrüchen durch Coronaviren seit 2000 (Stand 03.04.2020)

\begin{tabular}{|c|c|c|c|}
\hline & $\begin{array}{l}\text { SARS (Severe Acute Respiratory } \\
\text { Syndrome) }\end{array}$ & $\begin{array}{l}\text { MERS (Middle East respiratory } \\
\text { syndrome) }\end{array}$ & COVID-19 \\
\hline Ausbruch & 2002 bis $2004^{1}$ & $2012-\ldots$ & $2019-\ldots$ \\
\hline Erreger & SARS-CoV & MERS-CoV & SARS-CoV-2 \\
\hline Inkubationszeit & 2 bis 10 Tage; Mittel: 5 Tage & 7 bis 14 Tage & 1 -14 Tage; Mittel: 5 Tage \\
\hline Wirtstier (vermutlich) & Fledermäuse & Dromedare & Fledermäuse? Katzen? \\
\hline Übertragungsweg & $\begin{array}{l}\text { Tröpfchen } \\
\text { Schmierinfektion? }\end{array}$ & Tröpfchen? & $\begin{array}{l}\text { Tröpfchen } \\
\text { Schmierinfektion? }\end{array}$ \\
\hline Ursprung & China & Arabische Halbinsel & China \\
\hline Länder & 29 & 27 & 186 \\
\hline Gemeldete Fälle ${ }^{2}$ & $8.096^{3}$ (12/2003 WHO) & $2.499(12 / 2019 \mathrm{WHO})^{1}$ & $\begin{array}{l}103.228^{4} \text { (Deutschland) } \\
1.395^{5} \text { (weltweit) }\end{array}$ \\
\hline Todesfälle & 774 (са. 9\%) & 861 (са. 34\%) & $\begin{array}{l}1.861 \text { (Deutschland ca. } 0,3 \% \text { ), } \\
81.580 \text { (in } 206 \text { Ländern; weltweit ca. 4\%) }\end{array}$ \\
\hline Anmerkungen & Ausbruch wurde 2004 als beendet erklärt & $\begin{array}{l}\text { fast ausschließlich auf Saudi-Arabien } \\
\text { beschränkt }\end{array}$ & sehr schnelle Ausbreitung weltweit \\
\hline \multicolumn{4}{|c|}{$\begin{array}{l}\text { 'Erste Reporte 02/2003, Erkrankungen konnten aber nachträglich in } 2002 \text { zurückverfolgt werden; }{ }^{2} \text { Abruf: } 23.03 .2020 \text { : niaid.nih.gov/diseases-conditions/covid-19 } \\
{ }^{3} \text { Abruf: 23.03.2020: who.int/csr/sars/country/table2004_04_21/en/; }{ }^{4} \text { Abruf 09.04.2020: Täglicher Lagebericht des RKI zur Coronavirus-Krankheit-2019 vom 08.04; } \\
{ }^{5} \text { Abruf 09.04.2020: https://who.sprinkIr.com/ }\end{array}$} \\
\hline
\end{tabular}

keine Immunsupprimierten Patienten, keine MRSA-Patienten)

- Risiken durch Klimaanlagen (raumlufttechnische Anlagen) prüfen und minimieren

- Ambulanzen, Funktionsabteilungen: Räumliche und organisatorische Trennung bei der Versorgung von Verdachts- und bestätigten Fällen

\section{Mitarbeiterschutz:}

- Mitarbeiter schulen und (nach Möglichkeit) Freistellen von der Versorgung anderer Patienten

_ Persönliche Schutzausrüstung wie Schutzkittel, Einmalschutzhandschuhe, Atemschutzmaske FFP2 oder FFP3 (bei bestätigten Fällen),sonst Mund-Nasen-Schutz, Schutzbrille

- Schutzausrüstung verbleibt im Zimmer bzw. Schleuse, Einmalmaterial vor Verlassen des Zimmers bzw. der Schleuse in einem geschlossenen Behälter entsorgen

- Händedesinfektion nach Ausziehen der Schutzhandschuhe

- Beobachtung der Mitarbeitergesundheit

\section{Desinfektion und Reinigung:}

- Geeignete Desinfektionsmittel sollten VAH-gelistet sein und müssen mindestens „begrenzt viruzid“ gegen behüllte Viren, „begrenzt viruzid PLUS“ oder „viruzid“ als Kennzeichnung tragen. Bei behördlich angeordneter Desinfektion sind RKIgelistete Desinfektionsmittel zu verwenden. Aktuell gelten Ausnahmeregelungen für entsprechende in Apotheken hergestellte Desinfektionsmittel.

— Flächendesinfektion: Wischdesinfektion mind. 1x täglich von patientennahen Oberflächen (z.B. Nachttisch, Türgriffe, Nasszelle) und ggf. weiteren gefährdeten oder kontaminierten Oberflächen

- Medizinprodukte: Patientenbezogen verwenden und nach Gebrauch desinfizieren (wenn möglich thermische Desinfektion, ansonsten Instrumentendesinfektionsmittel); zentrale Aufbereitung: nur wenn der Transport in einem geschlossenen Behälter erfolgt. Grundlage ist hier die KRINKO-Empfehlung zur Medizinprodukteaufbereitung.

_ Geschirr: Transport im geschlossenen Behälter, Reinigung in Spülmaschine

- Wäsche: desinfizierendes Verfahren gemäß RKI Liste; Taschentücher: nur Einwegtücher verwenden

- Betten und Matratzen: wischdesinfizierbare Überzüge

— Schlussdesinfektion mit mind. „begrenzt viruziden“ Mitteln

\section{Abfallentsorgung:}

_ Einrichtungen mit sporadischen Fällen: Direkt vor Ort in reißfesten, feuchtigkeitsbeständigen und dichten Behältnissen sammeln; nicht Umfüllen oder Sortieren; in sicher verschlossenen Behältnissen (ggf. auch Rücklaufbehälter) zur zentralen Sammelstelle transportieren; bei Flüssigkeiten geeignetes Saugmaterial verwenden.

- Schwerpunktversorgung (z.B. Isolierstationen): Nach besonderen Vorgaben, gewöhnlich in Absprache mit dem Krankenhaushygieniker und örtlichen Behörden

\section{Umgang mit Verstorbenen:}

_ Leichname gelten als infektiös, daher sollte der Umgang nach 
den Regeln des Arbeitsschutzes erfolgen und der Kontakt mit Sekreten vermieden werden (s. aktuelle Hinweise des RKI)

\section{Patienten-Transport innerhalb der Einrichtung:}

- Möglichst vermeiden, sonst Ziel informieren, Einzeltransport

- Patient: Mund-Nasen-Schutz; beteiligte Mitarbeiter: Schutzkittel, Atemschutzmaske (FFP2), Schutzkittel, ggf. Schutzbrille; weitere Kontakte vermeiden

- Nach Abschluss der Untersuchung oder des Eingriffs: Flächen und Transportmittel ordnungsgemäß desinfizieren

\section{Patienten-Transport außerhalb der Einrichtung (z.B. Verlegung):}

_ Zieleinrichtung (z.B. Krankenhaus) vor Transportbeginn informieren

- Patient: Mund-Nasen-Schutz; beteiligte Mitarbeiter: Schutzkittel, Atemschutzmaske (FFP2), Schutzkittel, ggf. Schutzbrille; weitere Kontakte vermeiden; Transportende: unmittelbar alle Flächen und Gegenstände ordnungsgemäß desinfizieren

\section{Besucherregelung}

_ Besucherstopp oder -einschränkung nach aktuellen Vorgaben; Unterweisung der Besucher zu Sicherheitsmaßnahmen Als Grundlage für alle Hygienemaßnahmen gelten folgende KRINKO-Empfehlungen: Infektionsprävention im Rahmen der Pflege und Behandlung von Patienten mit übertragbaren Krankheiten (Bundesgesundheitsbl (2015) 58:1151-1170), Händehygiene in Einrichtungen des Gesundheitswesens (Bundesgesundheitsbl 2016 | 59:1189-1220) und die Anforderungen an die Hygiene bei der Aufbereitung von Medizinprodukten (Bundesgesundheitsbl 2012 | 55:1244-1310) sowie die Abfallentsorgungsrichtlinien der LAGA Nr. 18 und die TRBA 250.

Dr. Andrea Jessen

\section{PFLEGE EINFACH MACHEN}

COVID-19 verbreitet sich rasant und weist hohe Erkrankungszahlen auf.

Bei der Übertragung des SARS-CoV-2 spielen die oberen Atemwege eine eminent wichtige Rolle. Meist erfolgt die Übertragung direkt über Atemwegsekrete.

Da es bisher keine wirksame Therapie oder Impfung gibt und die Behandlung symptomatisch erfolgt, ist Übertragungsvermeidung besonders wichtig.

Aufgrund der dynamischen Entwicklungen müssen die aktuellen Empfehlungen des RKI beachtet werden.

\section{Sterillium ${ }^{\circledR}$-Produkte}

$\checkmark$ Sind wirksam gegen Corona-Viren

$\checkmark$ Besonders hautverträglich

$\checkmark$ Erhöhen die Hautfeuchtigkeit bei regelmäßiger Anwendung*

\section{Geeignete BODE-Desinfektionsmittel} gegen Corona-Viren:

- Hände: Sterillium-Produkte

- Haut: Cutasept-Produkte

- Oberflächen: Bacillol-, Kohrsolin-, Mikrobac- und Dismozon-Produkte

- Instrumente: Bomix plus und Korsolex-Produkte 


\title{
Erhöhtes Risiko bei COVID-19: Alter, Vorerkrankung, Demenz
}

\author{
Umgang mit Risikogruppen Es gibt keinen Teil der Bevölkerung, der nicht \\ von Corona betroffen sein kann. Aber einige Gruppen sind stärker gefährdet. \\ Dazu gehören neben Personen mit geschwächter Immunabwehr oder einge- \\ schränkter Lungenfunktion insbesondere aber Menschen mit Demenz. Was \\ sollten Pflegende beim Umgang mit Risikogruppen beachten?
}

$\mathbf{Z}$ u den Risikogruppen einer COVID-19-Infektion zählen Personen mit geschwächter Immunabwehr (z.B. Patienten mit malignen Erkrankungen und Chemotherapie, Patienten unter immunsuppressiver Therapie nach Transplantation) und Patienten unter Kortisontherapie (z.B. bei rheumatischen Erkrankungen, Lupus erythematodes). Auch Menschen mit angeborener oder erworbener Immunschwäche und ältere Menschen sind gefährdet. Im Alter wird das Immunsystem schwächer und häufig liegen internistische Erkrankungen vor. Besonders schwere Verläufe kann es geben bei Patienten mit eingeschränkter Lungenfunktion (z.B. Raucher, Patienten mit Asthma, COPD) und bei Patienten unter einer Glukokortikoidtherapie. Auch Diabetiker und Menschen mit chronischen Lebererkrankungen gehören dazu. Momentan gibt es folgende Erkenntnisse::

Asthma-Patienten: Aktuelle Empfehlungen der Fachgesellschaften raten zu einem Fortführen der individuell angepassten Inhalationstherapie, da es durch abruptes Absetzen zu einer Verschlechterung der Symptomatik kommen kann, was zu vermehrten Arztund ggf. Krankenhausaufenthalten führt, die wiederum das Infektionsrisiko erhöhen.

Schmerzpatienten: Meldungen, dass Ibuprofen den Krankheitsverlauf verschlimmern könnte, konnten bisher nicht wissenschaftlich bestätigt werden. Die nationalen Behandlungsrichtlinien empfehlen allerdings Paracetamol als Mittel der (ersten) Wahl bei Fieber oder Schmerzen.

Herz-Kreislauferkrankungen: Jede Infektion ist grundsätzlich eine zusätzliche Anstrengung für Herz und Kreislauf. Zusätzlich kommt es im Rahmen der COVID-19 bei etwa jedem sechsten stationär behandelten Patienten zu Herzrhythmusstörungen und in seltenen Fällen auch zu Herzmuskelschädigungen. Bei Patienten mit Herzinsuffizienz muss die Flüssigkeitsbilanzierung sorgfältig kontrolliert werden, da fiebrige Erkrankung eine erhöhte Flüssigkeitszufuhr erfordern, was unkontrolliert zur Dekompensation führen kann.
Schwangerschaft, Entbindung, Stillen: Derzeit gibt es keinen Anhalt, dass gesunde Schwangere ein höheres Risiko für eine Erkrankung oder schweren Verlauf haben. Ebenso gibt es bisher keine Hinweise auf eine Übertragung des Virus auf das Ungeborene im letzten Schwangerschaftstrimester. Dagegen wird vor Corona-bedingten Kaiserschnitten gewarnt, da diese als Eingriff mit einem allgemein höheren Komplikationsrisiko als die natürliche Entbindung für die Frauen einhergehen. Nach aktueller Datenlage geht man davon aus, dass auch keine Übertragung durch Muttermilch stattfindet.

\section{Umgang mit Menschen mit Demenz}

Demenz als solche ist zwar kein erhöhtes Risiko für eine Infektion oder schwerwiegenden Verlauf, jedoch leiden viele Betroffene unter Begleiterkrankungen, können durch Mangelernährung infektanfälliger sein und haben häufig Probleme, die notwendigen Maßnahmen der Händehygiene oder Atemetikette umzusetzen.

Aufgrund ihrer Erkrankung haben Menschen mit Demenz $(\mathrm{MmD})$ außerdem wenig Kompensationsstrategien, um mit veränderten Situationen und Abläufen zurechtzukommen. So können geänderte Besucherregelungen, der Wegfall von Gruppenaktivitäten und gemeinsamen Mahlzeiten, fehlende Ausflüge oder Spaziergänge eine akute Verschlechterung der Demenz-Symptomatik auslösen. Dies kann sich mit zunehmender Verwirrtheit, gesteigerter Unruhe oder aggressivem Verhalten äußern, was wiederum eine vermehrte Belastung der Mitarbeiter mit sich bringt. Aber auch ambulant versorgte Demenzkranke sind durch das Fehlen ihrer Betreuungsperson oder der Schließung von Tageseinrichtungen betroffen. Durch den daraus resultierenden akuten Versorgungsmangel werden Betroffene zum Teil temporär in neuer Umgebung oder von nicht vertrauten Personen versorgt. Doch gerade Vertrautheit, Regelmäßigkeit und Gewohnheit geben den Betroffenen das notwendige Schutzgefühl und Stabilität - eine besondere Herausforderung für die Pflegenden. Zu beachten ist:

Erklärung der Situation: Die aktuelle Pandemiesituation ist für $\mathrm{MmD}$ eine abstrakte Information, die sie nicht einordnen können, 
da insbesondere das Kurzzeitgedächtnis und Verarbeitung neuer Informationen früh verloren gehen. Nachrichten, die nicht emotional behaftet sind und vom Betroffenen nicht selbst durchlebt werden, finden dabei noch weniger Haftung. Hier kann es hilfreich sein, die Situation geduldig mehrfach zu erklären, sofern der Betroffene danach fragt. Dazu sollten einfache und kurze Sätze und nach Möglichkeit auch die gleichen Formulierungen verwendet werden.

\section{- Menschen mit Demenz haben wenig Kompensationsstrategien, um mit veränderten Situationen und Abläufen zurechtzukommen.}

Erinnerung: Die Wichtigkeit der Hände- und Hustenhygiene erfordert für Demenzerkrankte häufige, am besten visualisierte Erinnerungshilfen. Je nach Ausmaß der Erkrankung können Hinweisplakate über dem Waschbecken angebracht werden, die an das Händewaschen und die Dauer erinnern. Allerdings geht das Zeitgefühl im Rahmen einer Demenzerkrankung bereits relativ früh verloren, sodass „20 Sekunden“ wenig Bedeutung für die Betroffenen haben. Daher sollte in fortgeschrittenen Stadien die betreuende Person die gründliche Händehygiene immer wieder demonstrieren und begleiten.

Eine weitere Möglichkeit wäre, auf den jeweiligen Körperteil, der Aufmerksamkeit benötigt, in diesem Fall also die Hand zu zeigen oder leicht zu berühren. Wenn auch dies nicht mehr genügt, kann die sogenannte Hand-unter-Hand-Strategie angewendet werden, die den Betroffenen weitestgehend in der Ausführung von Handlungen unterstützt, ohne sie vollständig zu übernehmen. Je nach Ressourcen kann auch der Einsatz eines akustischen Signals oder von Melodien getestet werden.

Die Händehygiene kann langfristig trainiert und nach den Beschränkungen beibehalten werden, da sie auch hilft, andere Infektionserkrankungen wie Influenza zu vermeiden. Zudem ist es für $\mathrm{MmD}$ einfacher, Gewohnheiten langfristig beizubehalten.

Beobachtung: Die Verschlechterung aufgrund ungewohnter Umstände kann in vielen Fällen zeitverzögert nach einigen Tagen auftreten. Andererseits können Patienten im fortgeschrittenen Stadium einer Demenz ein bestehendes Krankheitsgefühl häufig nicht mehr verbal adäquat ausdrücken. Daher kommen der Patientenbeobachtung und Vitalzeichenkontrolle eine besondere Bedeutung zu. Frühe Zeichen für eine beginnende COVID-19-Erkrankung können rapide Verschlechterung des Verwirrtheitsgrades, Fieber, Husten, Kurzatmigkeit oder ungewöhnliche Abgeschlagenheit sein.

Persönliche Kontakte: Solange eine Kontaktbeschränkung gilt, können je nach individuellen Gegebenheiten Telefon und Videotelefonie zur Kontaktaufnahme mit Familienangehörigen eingesetzt werden. Wann und wo möglich, sollte die Kontaktaufnahme zu den Uhrzeiten der sonstigen Besuche stattfinden. Doch auch hierbei sollten die Hygienegrundlagen beachtet werden: soweit möglich personengebundene Gadgets (Geräte) verwenden, die im Zimmer verbleiben; Geräte regelmäßig desinfizieren.

Bewegungsdrang: Die bestehenden Ausgangseinschränkungen können zu einer gesteigerten Unruhe bei an Demenz Erkrankten führen. Hier können Aktivierungsübungen oder einfache Gymnastikübungen die Unruhe mildern, gegebenenfalls können ähnliche Aktivitäten, die sonst in der Gruppe durchgeführt werden, auch im Zimmer stattfinden. Die Alzheimer Gesellschaft hat Anregungen in ihrer kostenlosen App „Alzheimer \& YOU - den Alltag aktiv gestalten“ zur Verfügung gestellt (iPhone und Android).

Rituale: Das Gleichmaß der Abläufe mag Gesunden langweilig vorkommen, die Ritualisierung gibt den $\mathrm{MmD}$ jedoch Orientierung und Sicherheit. Daher sollten Gewohnheiten wie Mahlzeiten, Schlafengehen nach Möglichkeit zu den üblichen Zeiten stattfinden.

Dr. Andrea Jessen

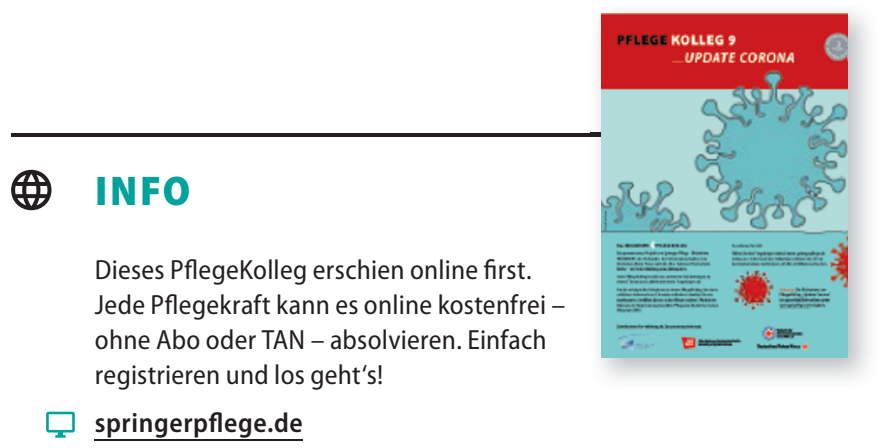

! PFLEGE EINFACH MACHEN

\begin{abstract}
Alle Menschen mit einem eingeschränkten Immunsystem oder Lungenfunktion gehören zu den Risikogruppen einer COVID-19 Erkrankung.
\end{abstract}

Eine besondere Gruppe sind Menschen mit Demenzerkran kungen. Da sie zu den vulnerablen Gruppen gehören, ist die Einhaltung der Händehygiene und Atemetikette besonders wichtig. Aufgrund ihrer Erkrankung sind sie außerdem in besonderem Maße auf Rituale und Gleichmäßigkeit der Abläufe angewiesen.

Veränderungen, auch eine mögliche Infektion, können sich in einer Verschlechterung der Demenz-Symptomatik äußern. Daher ist eine gründliche Patientenbeobachtung wichtig. Als Ausgleich für Spaziergänge können Gymnastikübungen helfen, ebenso wie Videotelefonie mit Angehörigen während der Besuchsverbote. 


\section{Fragebogen: Update Corona}

Es ist jeweils nur eine Antwort richtig.

1. Wenn Viruspartikel einmal in den Körper gelangt sind, dringen sie über bestimmte Oberflächenmoleküle in die Zielzellen ein. Wie nennt man das Prinzip, nach dem das geschieht?

A Schlüssel-Schloss-Prinzip

B Sender-Empfänger-Prinzip

C Ursache-Wirkung-Prinzip

2. Von welcher Inkubationszeit wird bei COVID-19 ausgegangen?

A Die Inkubationszeit liegt bei höchstens fünf Tagen.

B Es wird von ein bis 14 Tagen berichtet, wobei die meisten Patienten nach etwa fünf Tagen die ersten Symptome zeigen.

C Ausgegangen wird von 20 Tagen.

3. Im Zusammenhang mit der Pandemie taucht der Begriff Reproduktionszahl auf. Wofür steht er?

A Für die Zahl der Zweitinfektionen, die von einem Fall ausgehen.

B Er gibt an, wie viele Kinder ein während der Pandemie neugeborenes Mädchen durchschnittlich im Laufe seines Lebens hat.

C Für die Zeit, in der sich ein Corona-Virus vermehrt.

4. Für welche Erkrankungen außer Covid-19 waren Coronaviren ursächlich?
A SARS

B Ebola

C HIV

5. Was zählt, neben Fieber, Husten, Erschöpfung und Erkältungszeichen, zu häufigen Symptomen bei COVID-19?

A Geruchs- und Geschmacksverlust

B Juckreiz

C Starke Kopfschmerzen und Erbrechen

6. Welche Kennzeichnung müssen Desinfektionsmittel haben, die bei Covid-19 eingesetzt werden?

A Sie sollten als "fungizid" und "sporizid" gekennzeichnet sein.

B Die notwendige Deklaration ist "leuvurozid" und "bakterizid".

C Sie müssen mindestens „begrenzt viruzid“ gegen behüllte Viren, „, begrenzt viruzid PLUS" oder "viruzid" als Kennzeichnung tragen.

7. Worauf muss bei der Abfallentsorgung im COVID-19-Fall geachtet werden?

A Da gibt es keine besonderen Vorschriften.

B Abfälle müssen direkt vor Ort in reißfesten, feuchtigkeitsbeständigen und dichten Behältnissen gesammelt, in sicher verschlossenen Behältnissen zur zentralen Sammelstelle transportiert werden.
C Ausschließlich speziell geschulte Hygienefachkräfte entsorgen die Abfälle.

8. Welche Bevölkerungsgruppe gehört nicht zu den Risikogruppen in der Coronakrise?

A Personen mit geschwächter Immunabwehr

B Schwangere

C Patienten unter Chemotherapie

9. Warum bedeutet Demenz ein erhöhtes Risiko für eine SARS-CoV-2-Infektion?

A Weil Demenz als solche ein erhöhtes Risiko für eine Infektion darstellt.

B Weil von Demenz Betroffene oft unter Begleiterkrankungen leiden und Probleme haben, die notwendigen Maßnahmen der Händehygiene umzusetzen.

C Weil an Demenz Erkrankte starken Bewegungsdrang haben und oft anderen, z.B. beim eat by walking, begegnen.

10. Mit welcher Methode erinnert man an Demenz Erkrankte an die Hände- und Hustenhygiene?

A Geeignet sind häufige, am besten visualisierte Erinnerungshilfen.

B Telefonische oder Video-telefonische Erinnerungen durch Familienangehörige.

C Es gibt keine geeigneten Methoden, da das Kurzzeitgedächtnis nicht funktioniert.

\section{Ð ES IST JEWEILS NUR EINE ANTWORT RICHTIG.}

Mit dem HEILBERUFE PflegeKolleg können sich alle Pflegekräfte fortbilden. Wenn Sie 9 der 10 Fragen richtig beantworten, erhalten Sie ein anerkanntes Zertifikat, das Ihnen 3 Punkte im Rahmen der Registrierung beruflich Pflegender (RbP - www.regbp.de) beim Deutschen Pflegerat (DPR) sichert.

So nehmen Sie teil: Füllen Sie den Fragebogen unter springerpflege.de online aus. Unmittelbar nach der Teilnahme erfahren Sie, ob Sie bestanden haben und können sich Ihr Zertifikat gleich ausdrucken.
Die Online-Teilnahme ist für Abonnenten der Zeitschriften PROCARE und HEILBERUFE prinzipiell kostenlos.

Das PflegeKolleg „Update Corona“ haben wir für alle Pflegenden freigeschaltet. Sie brauchen kein Abo und keine TAN, um daran teilzunehmen.

Teilnahmeschluss ist der 27. November 2020.

$\square$ Die Auflösungen der abgelaufenen PflegeKollegs finden Sie auf springerpflege.de

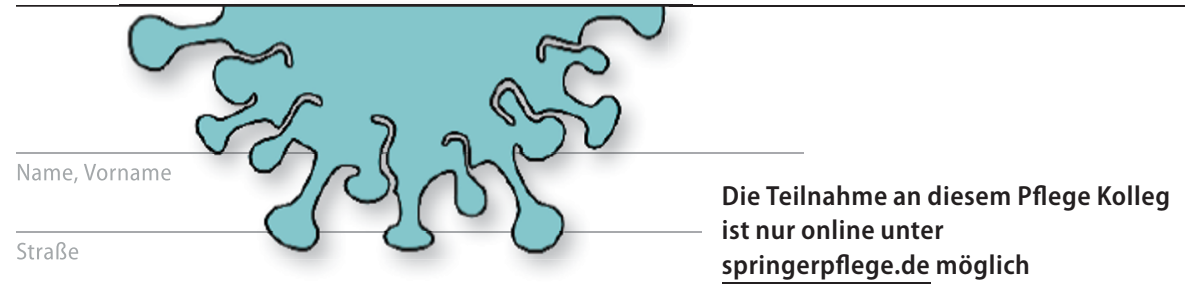

PLZ/Ort springerpflege.de möglich
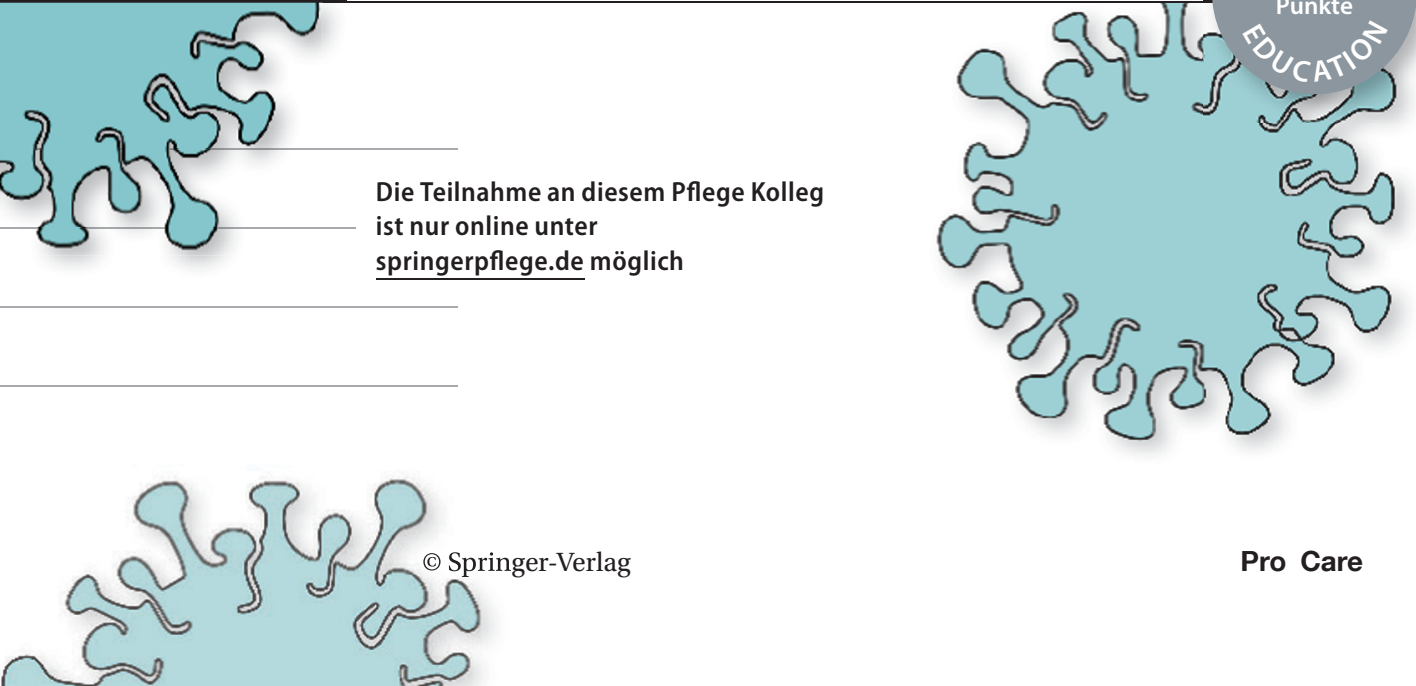